\title{
Transient excitons at metal surfaces
}

\author{
Xuefeng Cui ${ }^{1}$, Cong Wang', Adam Argondizzo', Sean Garrett-Roe ${ }^{2}$, Branko Gumhalter ${ }^{3}$ \\ and Hrvoje Petek ${ }^{1 \star}$
}

Excitons, electron-hole pairs bound by the Coulomb potential, are the fundamental quasiparticles of coherent light-matter interaction relevant for processes such as photosynthesis and optoelectronics $^{1-5}$. The existence of excitons in semiconductors is well established ${ }^{2}$. For metals, however, although implied by the quantum theory of the optical response, experimental manifestations of excitons are tenuous owing to screening of the Coulomb interaction taking place on timescales of a few femtoseconds $s^{6-8}$. Here we present direct evidence for the dominant transient excitonic response at a $\mathrm{Ag}(111)$ surface, which precedes the full onset of screening of the Coulomb interaction, in the course of a three-photon photoemission process with $\sim 15$ fs laser pulses. During this transient regime, electron-hole pair Coulomb interactions introduce coherent quasiparticle correlations beyond the single-particle description of the optics of metals that dominate the multi-photon photoemission process on the timescale of screening at a Ag(111) surface.

Reflection of light has made metal mirrors valued optical instruments since the Bronze Age ${ }^{9}$. At the macroscopic level the coherent optical response of a metallic surface is well described by the classical Maxwell's equations. At the quantum level, a photon interacting with a metal surface polarizes an electronhole $(e-h)$ pair to create an exciton-polariton, the quasiparticle of light-matter interactions ${ }^{10}$. The creation of excitons in insulators, semiconductors and molecules provokes many-body, coherent optical processes, which have been studied in the contexts of photosynthesis, vision, optical communication ${ }^{1-5}$, and so on. Yet, in metals the role of excitons remains uncharted, because the build up of screening on the timescale of plasma oscillations liberates bound states of the Coulomb potential ${ }^{6-8}$. The dynamical response of a metal surface to light terminates either in coherent reflection, or absorption, which can be detected through the photoelectric effect.

We reveal the transient optical response of the $\operatorname{Ag}(111)$ surface triggered by photoexcitation of an electron out of the occupied Shockley surface state (SS; Fig. 1) to form instantaneously a primary exciton composed of the electron interacting through the bare Coulomb interaction with its SS hole. In response, the retarded screening charge density fluctuations cause the bare Coulomb potential to wane, and simultaneously the image potential (IP) binding the electron to its screening image charge, to emerge as the asymptotic quasiparticle state. This transient regime of the coherent excitonic polarization is revealed through the intense excitonic local field, which augments the $m$ th-order multiphoton photoemission $(m \mathrm{PP})$ process.

The coherent optical response of metals has been studied by energy $(E)$ - and momentum $(k)$-resolved $m \mathrm{PP}$ spectroscopy. Interferometric time-resolved 2PP measurements have determined the surface and bulk hole dephasing times ${ }^{11,12}$. $2 \mathrm{PP}$ studies of silver

and copper surfaces have probed the electronic structure, dephasing and lifetimes of IP states ${ }^{13-16}$. Attosecond studies have revealed band-dependent photoelectron emission times ${ }^{17}$. The data were interpreted in terms of the external field exciting optical transitions between pre-existing electronic bands.

Excitons in metals have been more elusive. The excitonic response has been discussed in the context of theoretical models for the dielectric functions of metals ${ }^{18-20}$. Pertinently,
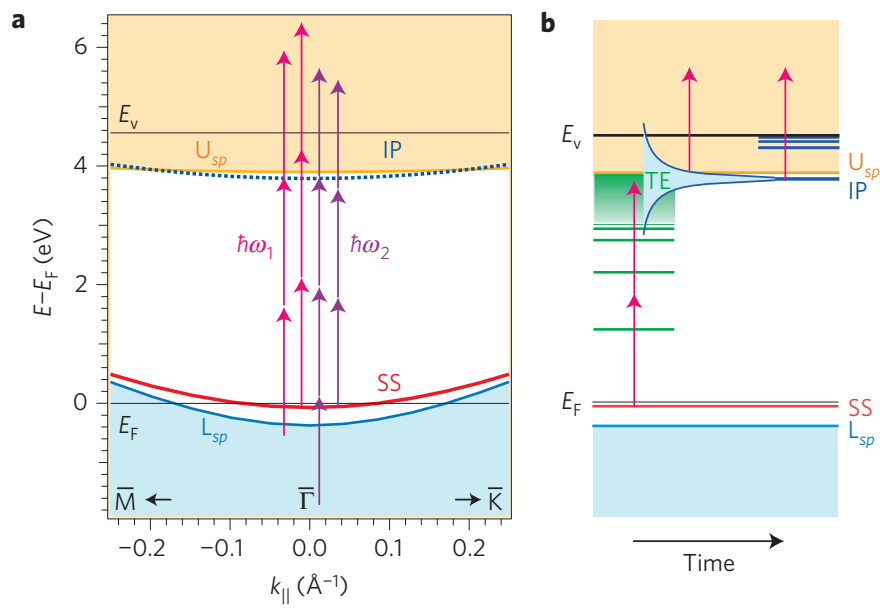

Figure 1 | The surface projected electronic band structure of $\mathrm{Ag}(111)$ and multiphoton photoemission processes at a $\mathrm{Ag}(111)$ surface. a, $\mathrm{A}$ band gap extends between -0.4 and $3.9 \mathrm{eV}$ from the lower to the upper $s p$-band $\left(L_{s p}\right.$ and $U_{s p}$ ) for the $\mathrm{Ag}(111)$ surface. Within the band gap, the Shockley surface state (SS; red line) and $n=1$ image potential state (IP; blue dotted line), with minima at -0.063 and $3.79 \mathrm{eV}$, form quantum wells at the metal-vacuum interface. The SS is occupied to $\left|k_{\|}\right|=0.07 \AA^{-1}$, where it intersects the Fermi level $\left(E_{F}\right)$. The $n=1 \mathrm{IP}$ state is the first member of a Rydberg-like series converging to the vacuum level ( $E_{\mathrm{v}}$; refs 16,25$)$. The vertical arrows indicate independent excitation pathways for 3PP and 4PP via the initial SS or the penultimate IP states for laser excitation wavelengths $\hbar \omega_{1}=2.20 \mathrm{eV}$ and $\hbar \omega_{2}=1.81 \mathrm{eV}$, corresponding to the spectra in Fig. 2a,h. b, Excitation scheme of IP states via the transient exciton (TE) manifold. Solving the Schrödinger equation for the bare Coulomb potential of the SS hole gives the eigenstates of the excitonic manifold (green lines); they converge in a quasi-continuum (shaded green) to the bottom of $U_{s p}$ (ref. 22). Charge density fluctuations cause the Coulomb field to evolve from that of the bare exciton to the IP state. The coherent 3PP measurements follow the time evolution of the polarization amplitude within the excitonic manifold towards the asymptotic IP state under the influence of time-dependent potentials and energy-time uncertainty, which is implied by the time-evolving width of the TE state. 

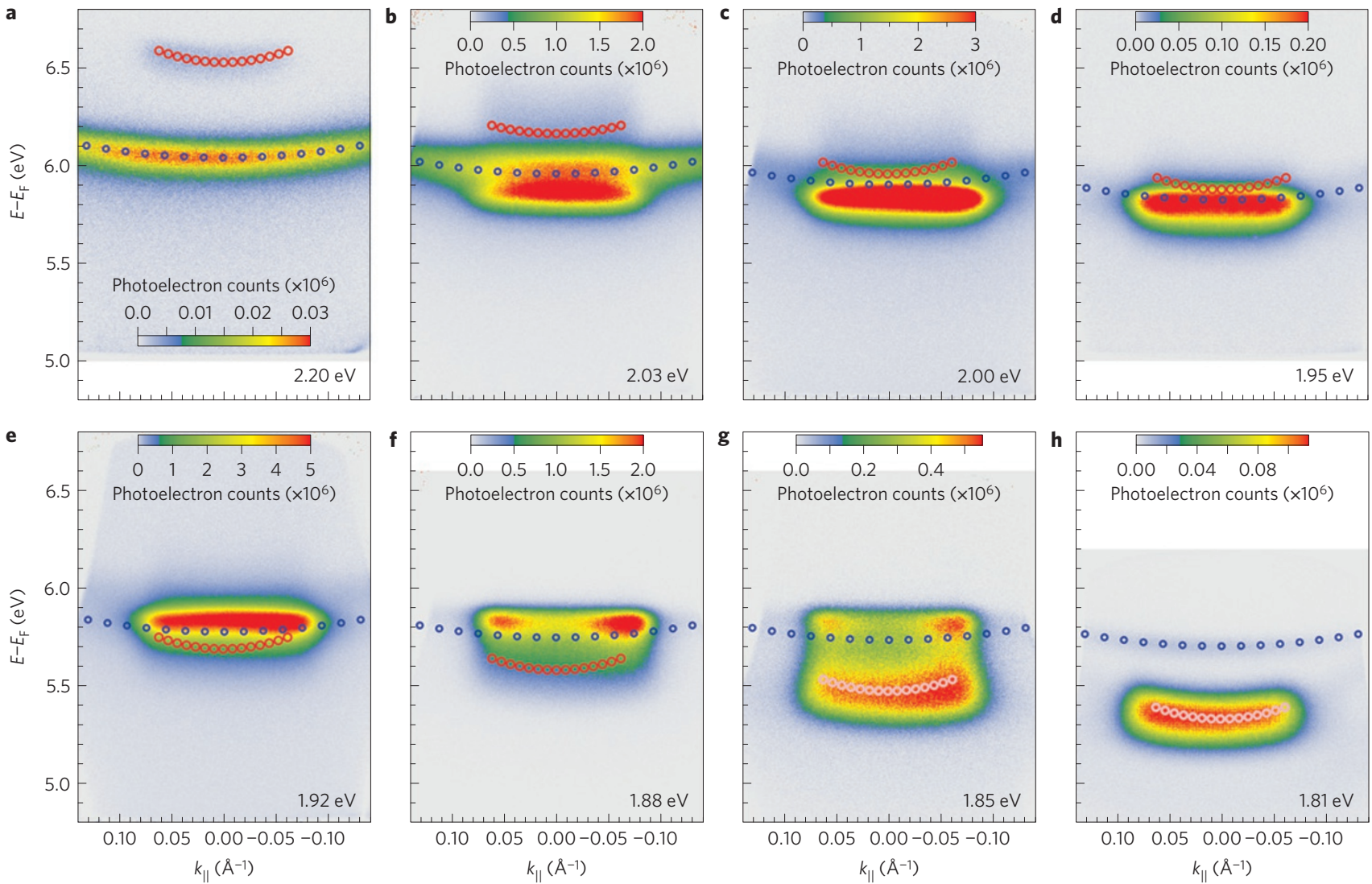

Figure 2 | 3PP and 4PP E $(k)$ distributions for photon excitation energies near the two-photon IP $\leftarrow$ SS resonance. a,h, For nonresonant excitation at $\hbar \omega_{\text {laser }}=1.81$ and $2.20 \mathrm{eV}$ the Shockley surface (SS) and image potential (IP) bands appear with $E(k)$ dispersions consistent with the band structure and the indicated excitation processes in Fig. 1a; the red and blue circles denote their expected parabolic dispersions. $\mathbf{b}-\mathbf{g}$, On tuning $\hbar \omega$ laser into the two-photon IP $\leftarrow$ SS resonance, a new feature characterized by enhancement of the transition moment, non-dispersive (that is, flat) $E(k)$ distribution spanning the occupied $k_{\|}$range of the SS, and photon-energy-independent photoelectron energy appears at $E_{f}=5.82 \pm 0.03 \mathrm{eV}$. We attribute these characteristics to a transient exciton created by excitation of an electron from the SS via the excitonic manifold, through a two-photon resonance involving the energy-conserving IP state. The photoelectron energies are given with respect to $E_{F}$. In all plots the white areas correspond to those for which there is no data.

the transient excitonic response has been proposed to have observable consequences in ultrafast $m$ PP spectroscopy ${ }^{21,22}$.

Here we present experimental evidence for the excitonic response in $m \mathrm{PP}$ spectra of the $\mathrm{Ag}(111)$ surface on excitation of a near-resonant two-photon IP $\leftarrow$ SS transition (Fig. 1) by intense, $\sim 15$ fs duration laser pulses, which has not been observed in onephoton resonant nanosecond laser experiments on $\operatorname{Ag}(111)$ and $\mathrm{Cu}(111)$ (refs 14,23). When the two-photon IP $\leftarrow$ SS resonance at $\hbar \omega_{\text {res }}=1.93 \pm 0.02 \mathrm{eV}$ lies outside the bandwidth of the excitation pulses, the SS and IP state spectra-that is, their energy versus momentum distributions $(E(k)$; Fig. 2a,h) in the $3 \mathrm{PP}$ spectra-appear as dispersive bands congruent with prior $2 \mathrm{PP}$ studies employing two-colour excitation ${ }^{16}$. A marked change in the $m \mathrm{PP}$ spectra arises when the laser spectrum subtends $\hbar \omega_{\text {res }}=1.93 \pm 0.02 \mathrm{eV}$ : a new spectral component appears at a constant final state energy $E_{\mathrm{f}}=5.82 \pm 0.03 \mathrm{eV}$ above the Fermi level $\left(E_{\mathrm{F}}\right)$. This new feature dominates the 3PP spectra (Fig. 2b-g), having an intensity more than 100 times greater than that of the nonresonantly excited SS and IP bands (Fig. 2a,h), it is nondispersive, and it does not exist in the single-particle band structure of the substrate (Fig. 1a); on the basis of these characteristics we argue for its assignment to a transient exciton (TE).

Plotting $E_{\mathrm{f}}$ for the SS, IP and TE states in Fig. 2 versus the excitation photon energy, $\hbar \omega_{\text {laser }}$, we find linear behaviour, with approximate slopes of three, one and zero (Fig. 3), which would be expected for a $3 \mathrm{PP}$ process if SS, IP and TE were the initial, penultimate and final states. Because no final state corresponding to TE exists at $E_{\mathrm{f}}=5.82 \mathrm{eV}$ (Fig. 1a; ref. 24), we search for its origin beyond the single-particle band structure.

An excitonic model has been previously elaborated for $2 \mathrm{PP}$ from the occupied Shockley states of $\mathrm{Ag}(111)$ or $\mathrm{Cu}(111)$ surfaces via the intermediate IP states $^{22}$, which approximately describes our experiment. Whereas the SS states are Bloch states confined to the surface by the boundary conditions, the IP states emerge through the retarded many-body screening response $\mathrm{e}^{25}$. There is no transition moment optically coupling the SS with the unformed IP state, so the excitation must involve the intermediary TE, which evolves into the IP state ${ }^{22}$. On creation of an exciton, charge density fluctuations respond to the Coulomb field to create the screened electron and hole quasiparticles. As the screening saturates, the IP state emerges as an on-the-energy-shell, asymptotically evolved excited surface quasiparticle $e^{22}$. The screening, however, is metal dependent: at a silver surface the quasiparticle correlation can persist for an $\sim 15$ fs timescale of dephasing of the low-frequency $(3.7 \mathrm{eV})$ surface plasmon, whereas at a copper surface it vanishes within $\sim 2$ fs (ref. 22 ).

The exciton on the $\operatorname{Ag}(111)$ surface forms by the interaction of an SS valence band hole with the upper $s p$-conduction-band $\left(U_{s p}\right)$ electron Bloch states (Fig. 1a) $)^{22,26}$. The wavefunction of the exciton, described by $N$-the quantum number for relative 
motion-and $\mathbf{K}$-the total centre-of-mass momentum wavevector-may be written as a superposition (wave packet) of the coupled hole $\mathbf{k}_{\mathbf{h}}$ and electron $\mathbf{k}_{\mathrm{e}}$ states $^{26}$,

$$
|\mathbf{K}, N\rangle=\sum_{\mathbf{k}_{e}, \mathbf{k}_{h}} \Psi_{\mathbf{k}_{e}, \mathbf{k}_{h}}^{\mathbf{K}, N}\left|\mathbf{k}_{e}, \mathbf{k}_{h}\right\rangle
$$

where $\Psi_{\mathbf{k}_{e}, \mathbf{k}_{h}}^{\mathrm{K}, N}$ is the amplitude of the constituent band states contributing to the exciton in the $k$-space, and $\mathbf{K} \approx 0$ is the momentum imparted by the photon ${ }^{26}$. The excitonic eigenenergies (Fig. 1b) are obtained by solving the Schrödinger equation for the bare Coulomb potential of the SS-hole charge density ${ }^{22}$.

The salient features of an exciton expressed in equation (1) directly explain two facets of the TE behaviour: the nondispersive TE photoemission derives from it being a localized superposition state in the relative coordinate space constrained by $\mathbf{K}=\mathbf{k}_{e}+\mathbf{k}_{h} \approx 0$; and its optical transition moment derives enhancement from the summation over the available interband transitions. Furthermore, the congruence of the $k_{\|}$ranges of the TE and SS attests to the TE being the superposition of all SS states within its $63 \mathrm{meV}$ occupied bandwidth.

To investigate the role of the TE precursor to the IP state, we image the photoelectron $E(k)$ distributions as a function of delay $\tau$ between identical, collinear, phase-correlated pump-probe pulses. Imaging $E(k)$ distributions with delay intervals of $\Delta \tau \sim 100$ as for $\hbar \omega_{\text {laser }}=2.05 \mathrm{eV}$, records a movie of the coherent polarization dynamics (Supplementary Movie 1). The interferograms for $k_{\|}=0$ and $E(k)$ distribution for $\tau=0$ (Fig. 4a,b) represent crosssections through the three-dimensional $(E, k, \tau)$ data. The distinct polarization dynamics of the SS, IP and TE are evident in the correlation traces (Fig. $4 \mathrm{c}-\mathrm{e}$ ) representing cross-sections through the interferogram for their respective $E_{\mathrm{f}} s$ marked by the lines cutting Fig. 4 a.

Instead of single-point correlations, however, we analyse the co-relationship between the SS, IP and TE polarizations by performing Fourier transforms of the interferograms for three representative values of $k_{\|}$. This produces 2D spectra representing the energy of linear polarization excited in the sample versus photoelectron energy relative to the SS band minimum (Fig. $4 \mathrm{f}-\mathrm{h}$ and Supplementary Fig. 1 give the complete 2D spectra, including the weaker nonlinear components). The $2 \mathrm{D}$ spectra reveal that, even for a detuning of the external field from the two-photon resonance, $\Delta=\hbar \omega_{\text {laser }}-\hbar \omega_{\text {res }}=0.12 \mathrm{eV}$, the energy of polarization driving the $3 \mathrm{PP}$ process is dominantly the local field at $\hbar \omega_{\text {res }}$, which excites the TE emission, rather than at $\hbar \omega_{\text {laser }}$, which excites the IP state emission (Fig. 4f,g). Whereas the external driving field varies spatially with the vacuum wavelength of light, the local field varies on the atomic scale ${ }^{27-29}$. Furthermore, the disposition and intensity of the TE and SS responses along the line with a slope of $1 / 3$ (Fig. 4f,g) shows that the coherent three-photon excitation of electrons from the SS is far more efficient by the local field than by the external field. The emission from the SS is distributed along this line in the 2D spectra between the dominant TE and the highenergy limiting SS features because an SS electron is photoemitted by absorption of a total of three photons from either the local $\hbar \omega_{\text {res }}$ or the external $\hbar \omega_{\text {laser }}$ fields.

Being retarded in time, the IP state cannot participate in a coherent $3 \mathrm{PP}$ process, and therefore its disposition within the $2 \mathrm{D}$ spectra is distinct (Fig. $4 \mathrm{f}-\mathrm{h}$ ). Because the $2 \mathrm{D}$ spectrum of the IP state is aligned with a slope of $1 / 2$ both within (Fig. 4f,g) and outside (Fig. 4h) the occupied $k_{\|}$range of the SS, we conclude that it is excited from the continuum of bulk bands belonging to the lower $s p$-band $\left(L_{s p}\right.$ : Fig. 1a) by absorption of two photons at $\hbar \omega_{\text {laser }}$ from the external field. The weak dependence of the IP state $2 \mathrm{D}$ spectra with $k_{\|}$, shown in Fig. $4 \mathrm{f}-\mathrm{h}$, indicates that, for $\Delta=0.12 \mathrm{eV}$, the $L_{s p}$ channel dominates the 3PP via the IP state, even when the TE channel

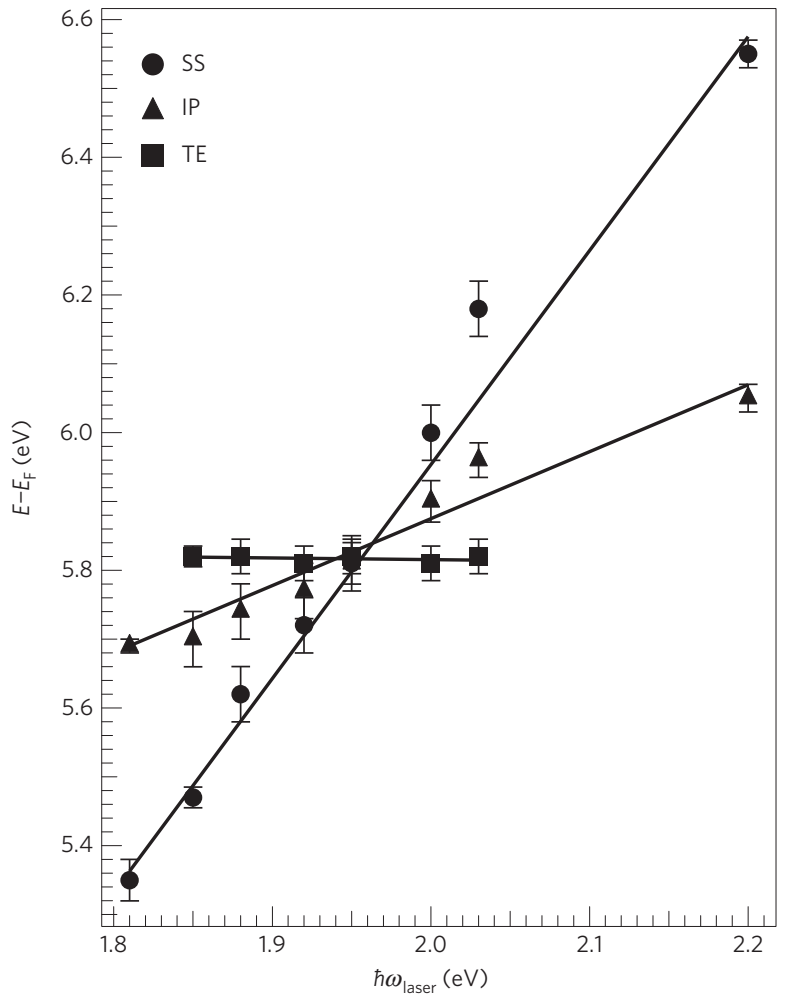

Figure 3 | Tuning of the spectroscopic features with photon energy. The photoelectron energies of the Shockley surface (SS), image potential (IP) and transient exciton (TE) states in Fig. 2 are plotted versus the excitation photon energy. Assuming that the photoelectron energy in multiphoton photoemission processes is defined by the photon energy, the tuning of $E_{\mathrm{f}}$ of a spectroscopic feature is determined by the number of photons times the photon energy required to excite an electron from the energy band to above $E_{\mathrm{v}}$. Therefore, in the 3PP process, the initial state (SS) is expected to tune with a slope of three and the penultimate state (IP) with a slope of one, as observed. Accordingly, a slope of zero would attribute the $T E$ feature to a sharply defined nondispersive state at $E_{f}=5.82 \pm 0.03 \mathrm{eV}$, which does not exist in the band structure of $\mathrm{Ag}$. The TE behaviour, however, can be explained by the existence of a local excitonic field, $\hbar \omega_{\text {res, }}$ associated with the IP $\leftarrow$ SS two-photon resonance, which is excited within the bandwidth of the broad, tunable excitation pulses. The error bars represent uncertainties in fitting of the spectral features to Gaussian lineshapes.

dominates the SS emission. The emergence of the IP state from the TE channel and its subsequent photoemission by a single-photon process, however, can be discerned from the additional density in the $2 \mathrm{D}$ spectra for $\left|k_{\|}\right|<0.07 \AA^{-1}$, which extends between TE and IP features along the line with slope of one (Fig. $4 \mathrm{f}, \mathrm{g}$ ). The presence of two channels for IP state photoemission via interactions with the $\hbar \omega_{\text {res }}$ and $\hbar \omega_{\text {laser }}$ fields leads to polarization beating in its correlation trace at $\tau \cong 20 \mathrm{fs}$ (Fig. $4 \mathrm{~d}$ ). A time-windowed frequency analysis of the interferogram in Fig. 4a shows that the IP state photoemission driven by $\hbar \omega_{\text {laser }}$ dominates for $<20 \mathrm{fs}$, to be overtaken by the more slowly dephasing TE channel, which the frequency upchirps from $\hbar \omega_{\text {res }}$ near $\tau=0$ towards $\hbar \omega_{\text {laser }}$ for $\tau>20 \mathrm{fs}$, as implied by the additional density between the TE and IP features in the 2D spectra. As $\Delta \rightarrow 0$, the creation of the IP state from the excitonic manifold becomes dominant, as is evident in Fig. 2. Model simulations of twopulse correlations (Supplementary Fig. 2), which reproduce the long dephasing time of the linear polarization, support the scenario in Fig. 1b, where the dense TE manifold is the precursor to the IP state under resonant conditions. 

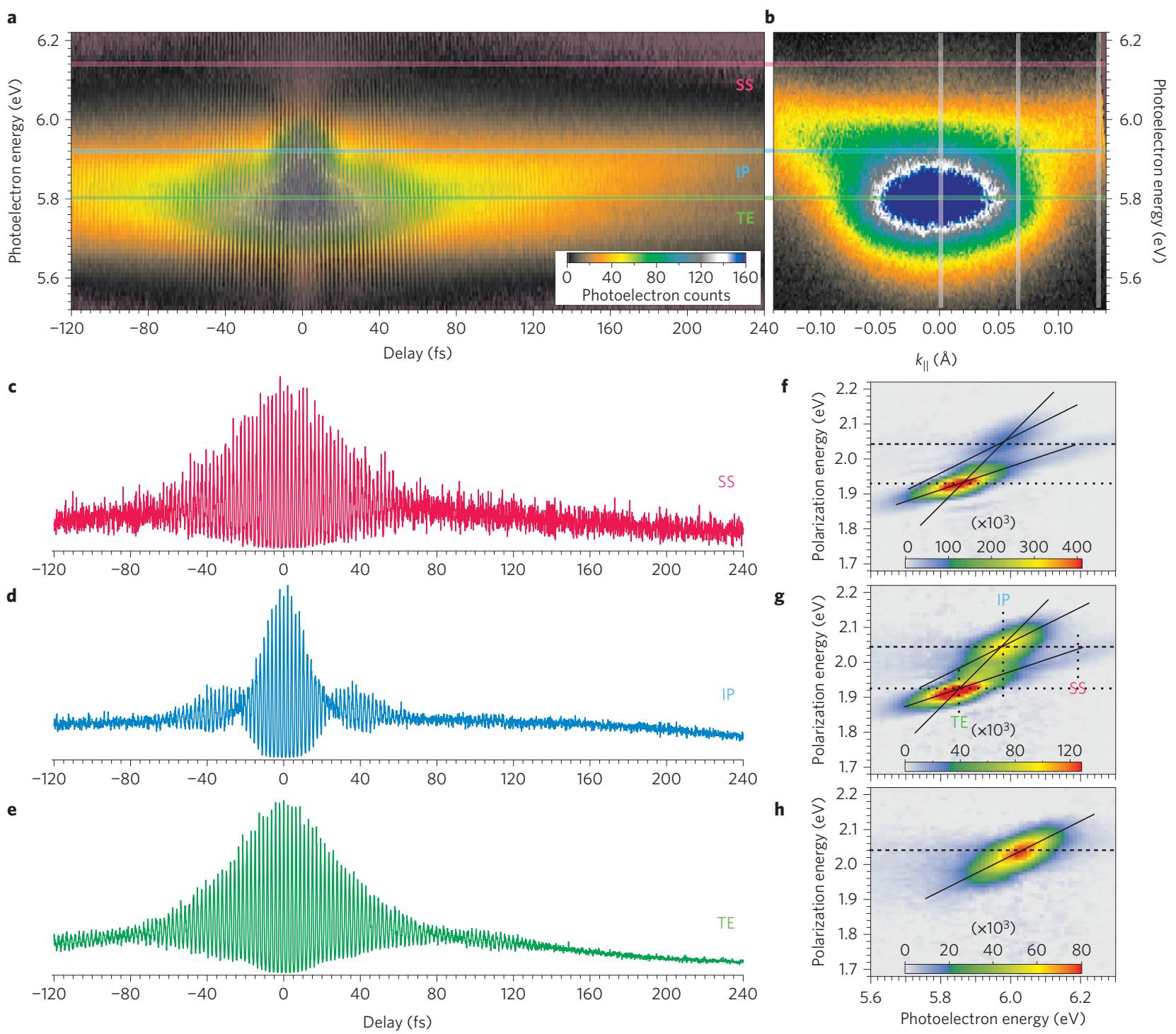

Figure 4 | 3D photoemission spectra for near-resonant two-photon IP $\leftarrow$ SS excitation. a, Interferogram of the photoelectron counts versus photoelectron energy and time delay between interferometrically scanned pump-probe pulses for $k_{\|}=0$. The laser energy $\hbar \omega$ laser $=2.05$ eV is detuned

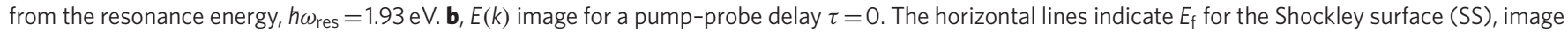
potential (IP) and transient exciton (TE) in c-e, and the vertical lines indicate $k_{\|}$for the $2 \mathrm{D}$ spectra in $\mathbf{f}-\mathbf{h}$. c-e, Correlation measurements ${ }^{11}$ obtained by taking cross-sections through the interferogram in a at $E_{f}$ for the SS, IP and TE. Oscillations with approximately the period of the carrier wave ( 2 fs) represent interference between the pump and probe pulse-induced polarizations excited in the sample. f-h, $2 \mathrm{D}$ photoelectron spectra obtained by Fourier transforming interferometric scans such as in a for $k_{\|}=0.0 \mathbf{f}, 0.067 \mathbf{g}$, and $0.134 \AA^{-1}$. h, The Fourier axis is labelled the 'polarization energy'. Higher-order polarization components are substantially weaker (Supplementary Fig. 1). The 2D plots show the correlation between the coherent polarization and

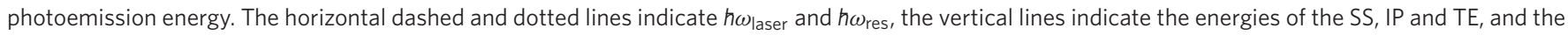
full lines designate slopes of $1 / 3,1 / 2$ and 1.

The 2D spectra also explain the excitonic origin of the constant TE photoemission energy. On femtosecond pulse illumination, an electron is excited by two-photon interaction from the SS through a manifold of excitonic states converging in a quasicontinuum to the bottom of $U_{s p}$ (Fig. 1b). Concurrently, the screening involving off-the-energy-shell transients causes the bare exciton to evolve into its saturated form, the uncorrelated IP electron and SS hole quasiparticles ${ }^{30}$. In the course of this quantum kinetic evolution, according to energy-time uncertainty, the bandwidth of the coherent polarization is determined by the interaction time $e^{31,32}$ : constructive and destructive interference causes different frequency components of the coherent polarization to grow if they conserve energy, or to die off if they do not. Therefore, during the interaction, the excitonic local field grows at $\hbar \omega_{\text {res }}$ because its dephasing time is slower than the pulse duration and it conserves energy at the two-photon resonance. Moreover, the local field is integrated over the duration of the external field pulse and is localized at the surface on the atomic scale, therefore the probability of an SS electron absorbing a photon from the local field is greater than from the external field ${ }^{28,33}$. TE photoemission appears as long as the external field has sufficient amplitude to excite the local field at $\hbar \omega_{\text {res }}$. Because the surface response determines the local field, the TE emission energy is pinned at $3 \times \hbar \omega_{\text {res }}$. The SS emission in Fig. 2 via three-photon absorption from the weaker $\hbar \omega_{\text {laser }}$, however, tracks the photon energy.

The correlated TE photoemission is a manifestation of the fundamental screening response of condensed matter to polarization by optical fields. In contrast to interference effects in 
linear and nonlinear photoemission spectra with pulse durations exceeding the screening timescale ${ }^{14,23,29}$, the dominant excitonic response in the transient screening regime completely overwhelms the single-particle band structure features. Although excitons are detected as stable quasiparticles in semiconductors and insulators, the dynamical dielectric response in their formation and propagation is universal. For example, the local field-induced multiple-quantum transient exciton interactions in silver may help explain multi-exciton generation in organic films ${ }^{1}$. The observation of strong $e-h$ correlations, even in a noble metal, by coherent $m \mathrm{PP}$ spectroscopy holds promise for studies of nonequilibrium quasiparticle dynamics in strongly correlated materials.

\section{Methods}

Femtosecond laser excitation. The photoexcitation source for the $m \mathrm{PP}$ measurements is a noncollinear parametric amplifier (NOPA) system pumped by a Clark MXR Impulse fibre-laser oscillator-amplifier system. The NOPA system is used with $80-100 \mathrm{~mW}$ average power at a $1.25 \mathrm{MHz}$ repetition rate and $\sim 15 \mathrm{fs}$ pulse duration. The p-polarized light incident at $45^{\circ}$ from the surface normal is focused onto the $\operatorname{Ag}(111)$ sample.

Interferometric pump-probe delay scanning. Identical pump-probe pulse replicas are generated in a self-made Mach-Zehnder interferometer (MZI; ref. 11). The pump-probe delay is scanned with a piezoelectrically actuated translation stage at a rate of $7.9 \mathrm{fs} \mathrm{s}^{-1}$. Recording interference fringes at the centre laser wavelength by passing the secondary output of the MZI through a monochromator, and recording the resulting interferogram with a photodiode, calibrates the delay scanning. After data acquisition, about 200 interferometric scans are combined in software using the calibration interference fringes.

Photoelectron imaging. The $m \mathrm{PP}$ photoelectron images are recorded with a Specs Phoibos 100 electron spectrometer equipped with a 3D-DLD delay-line photoelectron counting detector. For each interferometer pump-probe delay scan, $4,096 E(k)$ images are taken with an integration time of $12 \mathrm{~ms}$ per image.

Sample. The single-crystal $\mathrm{Ag}(111)$ surface is prepared by conventional surface science methods in an ultrahigh vacuum chamber with a base pressure of $<10^{-10}$ mbar. During the measurements the sample is cooled to $\sim 100 \mathrm{~K}$.

Received 31 December 2013; accepted 2 May 2014; published online 1 June 2014

\section{References}

1. Chan, W-L. et al. Observing the multiexciton state in singlet fission and ensuing ultrafast multielectron transfer. Science 334, 1541-1545 (2011).

2. Gibbs, H. M., Khitrova, G. \& Koch, S. W. Exciton-polariton light-semiconductor coupling effects. Nature Photon. 5, 273-273 (2011).

3. Turner, D. B. \& Nelson, K. A. Coherent measurements of high-order electronic correlations in quantum wells. Nature 466, 1089-1092 (2010).

4. Cundiff, S. T. \& Mukamel, S. Optical multidimensional coherent spectroscopy. Phys. Today 66, 44-49 (July, 2013).

5. Lee, H., Cheng, Y-C. \& Fleming, G. R. Coherence dynamics in photosynthesis: Protein protection of excitonic coherence. Science 316, 1462-1465 (2007).

6. Edwards, P. P., Lodge, M. T. J., Hensel, F. \& Redmer, R. '... a metal conducts and a non-metal doesn't'. Phil. Trans. R. Soc. A 368, 941-965 (2010).

7. Silkin, V. M., Kazansky, A. K., Chulkov, E. V. \& Echenique, P. M. Time-dependent screening of a point charge at a metal surface. J. Phys. Condens. Matter 22, 304013 (2010).

8. Huber, R. et al. How many-particle interactions develop after ultrafast excitation of an electron-hole plasma. Nature 414, 286-289 (2001).

9. Enoch, J. M. History of mirrors dating back 8000 years. Optom. Vis. Sci. 83, 775-781 (2006)

10. Hopfield, J. J. Theory of the contribution of excitons to the complex dielectric constant of crystals. Phys. Rev. 112, 1555-1567 (1958).

11. Ogawa, S., Nagano, H., Petek, H. \& Heberle, A. P. Optical dephasing in $\mathrm{Cu}(111)$ measured by interferometric two-photon time-resolved photoemission. Phys. Rev. Lett. 78, 1339-1342 (1997).

12. Petek, H., Nagano, H. \& Ogawa, S. Hole decoherence of $d$ bands in copper. Phys. Rev. Lett. 83, 832-835 (1999).
13. Güdde, J., Rohleder, M., Meier, T., Koch, S. W. \& Höfer, U. Time-resolved investigation of coherently controlled electric currents at a metal surface. Science 318, 1287-1291 (2007).

14. Giesen, K. et al. Image potential states seen via two-photon photoemission and second harmonic generation. Phys. Scr. 35, 578-581 (1987).

15. Schoenlein, R. W., Fujimoto, J. G., Eesley, G. L. \& Capehart, T. W. Femtosecond relaxation dynamics of image-potential states. Phys. Rev. B 43, 4688-4698 (1991).

16. Marks, M., Schwalb, C. H., Schubert, K., Güdde, J. \& Höfer, U. Quantum-beat spectroscopy of image-potential resonances. Phys. Rev. B 84, 245402 (2011).

17. Cavalieri, A. L. et al. Attosecond spectroscopy in condensed matter. Nature 449, 1029-1032 (2007).

18. Mueller, F. M. \& Phillips, J. C. Electronic spectrum of crystalline copper. Phys. Rev. 157, 600-607 (1967).

19. Fong, C. Y., Cohen, M. L., Zucca, R. R. L., Stokes, J. \& Shen, Y. R. Wavelength modulation spectrum of copper. Phys. Rev. Lett. 25, 1486-1490 (1970).

20. Marini, A. \& Del Sole, R. Dynamical excitonic effects in metals and semiconductors. Phys. Rev. Lett. 91, 176402 (2003).

21. Schöne, W-D. \& Ekardt, W. Transient excitonic states in noble metals and Al. Phys. Rev. B 65, 113112 (2002).

22. Gumhalter, B., Lazić, P. \& Došlić, N. Excitonic precursor states in ultrafast pump-probe spectroscopies of surface bands. Phys. Status Solidi (b) 247, 1907-1919 (2010).

23. Wallauer, W. \& Fauster, T. Two-photon excitation processes and linewidths of surface and image states on $\mathrm{Cu}(111)$. Surf. Sci. 374, 44-50 (1997).

24. Miller, T., Hansen, E. D., McMahon, W. E. \& Chiang, T. C. Direct transitions, indirect transitions, and surface photoemission in the prototypical system Ag(111). Surf. Sci. 376, 32-42 (1997).

25. Echenique, P. M. \& Pendry, J. B. Theory of image states at metal surfaces. Prog. Surf. Sci. 32, 111-159 (1990).

26. Elliott, R. J. Intensity of optical absorption by excitons. Phys. Rev. 108, 1384-1389 (1957).

27. Raseev, G. Plasmon resonances of $\mathrm{Ag}(001)$ and $\mathrm{Ag}(111)$ studied by power density absorption and photoyield. Surf. Sci. 615, 6-20 (2013).

28. Merschdorf, M., Kennerknecht, C. \& Pfeiffer, W. Collective and single-particle dynamics in time-resolved two-photon photoemission. Phys. Rev. B 70, 193401 (2004).

29. Samuelsen, D. \& Schattke, W. Photoemission by screened photon fields from layered solids. Surf. Sci. 327, 379-386 (1995).

30. Gumhalter, B. Stages of hot electron dynamics in multiexcitation processes at surfaces: General properties and benchmark examples. Prog. Surf. Sci. 87, 163-188 (2012).

31. Leitenstorfer, A. et al. Ultrafast coherent generation of hot electrons studied via band-to-acceptor luminescence in GaAs. Phys. Rev. Lett. 73, 1687-1690 (1994).

32. Petek, H. et al. Optical phase control of coherent electron dynamics in metals. Phys. Rev. Lett. 79, 4649-4652 (1997).

33. Winkelmann, A. et al. Resonant coherent three-photon photoemission from $\mathrm{Cu}(001)$. Phys. Rev. B 80, 155128 (2009).

\section{Acknowledgements}

This research was supported by Division of Chemical Sciences, Geosciences, and Biosciences, Office of Basic Energy Sciences of the US Department of Energy through Grant DE-FG02-09ER16056. The authors thank T. Kampen for help with the 3D-DLD detector and the data acquisition software.

\section{Author contributions}

H.P. conceived the experiment and wrote the first draft of the manuscript. X.C. and C.W discovered the transient exciton in $\mathrm{Ag}(111)$, set up the photoelectron imaging system, wrote the data acquisition and analysis programs, and performed all of the experiments and data analysis. A.A. set up the NOPA system and contributed to its operation. S.G-R. provided expertise in 2D electronic spectroscopy. B.G. predicted the TE phenomenon and performed the theoretical simulations. All co-authors contributed to the discussion and elucidation of the TE phenomenon.

\section{Additional information}

Supplementary information is available in the online version of the paper. Reprints and permissions information is available online at www.nature.com/reprints.

Correspondence and requests for materials should be addressed to H.P.

\section{Competing financial interests}

The authors declare no competing financial interests. 